\title{
Trenzar los hilos de la escuela. El liderazgo, elemento clave para el funcionamiento de los consejos técnicos escolares
}

\section{Braiding the threads of the school. Leadership a key element for the operation of Technical School Councils}

\section{LUIS FELIPE GÓMEZ*}

Los consejos técnicos escolares tienen ya algunos años en operación; sin embargo, hay pocos estudios que aborden su funcionamiento. El objetivo de este trabajo es analizar el liderazgo que ejercen profesores y director o directora en las sesiones de este órgano colegiado. El estudio se realizó en una secundaria pública de la ciudad de Guadalajara, a través de la metodología cualitativa aplicada a un estudio de caso. Mediante la observación de las sesiones del consejo durante el ciclo escolar 2016-2017 y diez entrevistas a profesores y al director, caracterizamos el liderazgo de los profesores que, aunque menos notorio, es tan importante como el del director, pues permite examinar información, generar documentos y tomar decisiones razonadas. En tanto, el director cumple las labores de fijar el rumbo, promover el desarrollo del personal y, en menor medida, transformar la escuela. Su tipo de liderazgo es predominantemente transformacional, pero también tiene rasgos democráticos y carismáticos; el de los profesores es horizontal, temporal, asignado por colegas o autoasignado. Concluimos que el liderazgo es uno de los elementos indispensables para el buen funcionamiento del Consejo Técnico Escolar; las características identificadas en este estudio pueden ser útiles para que otras escuelas aprendan y mejoren el funcionamiento del organismo colegiado encargado de promover una mayor autonomía de gestión en sus escuelas.

Despite the fact that the Technical School Councils have already been in operation for a few years, a small number of studies analyze their functioning. The purpose of this article is to analyze the leadership of teachers and the principal in the sessions of this collegiate body. The study was conducted in a public junior high school in Guadalajara. The methodology was a qualitative case study. Through the observation of the sessions of the Council, during the school year 2016-2017 and ten interviews with teachers and the principal, the leadership of the professors was characterized. Although less noticeable, it is as important as that of the principal, since it allows to analyze information, generate documents and make reasoned decisions. The article also describes the functions and type of leadership. The findings show that it fulfills the functions of setting the course, promoting the development of personnel and, to a lesser extent, transforming the school. The type of leadership is predominantly transformational, but also has traits of the democratic and charismatic; that of teachers is horizontal, temporary, assigned by colleagues, or self-assigned. The author concludes that leadership is one of the crucial elements for the proper functioning of the School Technical Council and that the characteristics identified in this case study can be useful for other schools to learn and improve the functioning of the collegiate body in charge of promoting greater management autonomy in their schools.

\section{Palabras clave:}

liderazgo pedagógico, Consejo Técnico Escolar, gestión escolar, autonomía escolar

\section{Keywords:}

pedagogical leadership, School Technical Council, school management, school autonomy

Recibido: 8 de junio de 2018. Aceptado para publicarse: 13 de diciembre de 2018. Recuperado de: https://sinectica.iteso.mx/index.php/SINECTICA/article/view/882 DOI: $10.31391 / S 2007-7033(2019) 0052-009$

* Doctor en Educación. Profesor e investigador del Departamento de Psicología, Educación y Salud del ITESO. Miembro del Sistema Nacional de Investigadores. Su área de especialidad son los procesos de enseñanza-aprendizaje y los procesos cognitivos. Correo electrónico: lgomez@iteso.mx/ http://www.investigadores.iteso.mx/detalleinv.php?id_inv=33 


\section{INTRODUCCIÓN}

A estudiar los elementos socioculturales que permiten el buen funcionamien$A$ to de los consejos técnicos escolares (CTE), encontramos que el liderazgo es 2 uno de ellos. Por tanto, en este artículo analizamos las funciones y los tipos de liderazgo que se ejercen durante las sesiones de trabajo de este organismo colegiado en una secundaria pública. Develar y presentar en forma explícita la manera en que se ejerce el liderazgo por parte de los docentes y el director servirá a otras escuelas para que analicen sus propios modos de proceder y, en su caso, aprender de lo que funciona en la citada escuela.

El estudio del funcionamiento de los CTE es relevante en virtud de que el objetivo central de este colectivo es que la escuela tenga una mayor autonomía de gestión con la participación de todos los actores escolares en la toma de decisiones (SEP, 2013). Si hay un resquicio que permita a las secundarias centrarse en atender las necesidades locales de estudiantes y profesores, es precisamente el CTE, pero para que logre su cometido, es indispensable el trabajo colaborativo, la participación comprometida de los profesores y una buena conducción del proceso.

El caso analizado es un ejemplo de buena práctica de trabajo en el CTE, dado que hay un esfuerzo concertado en el colectivo escolar para hacer planeaciones que respondan a los estudiantes de cada grupo, diversificar las maneras de enseñar e incrementar la asistencia a clases. A pesar de ser un buen ejemplo de trabajo colegiado, advertimos cierta tendencia de algunos profesores a no hacerse responsables de las dificultades que presentaban los alumnos y no considerar las fallas en que incurrían; en cambio, como parte del liderazgo directivo, observamos un esfuerzo por destacar la responsabilidad de la escuela respecto del aprendizaje de cada alumno, independientemente de sus condiciones familiares, económicas o neurológicas. No es un colectivo escolar excepcional, pero observamos un esfuerzo deliberado y consistente de la mayoría de los integrantes en lograr una mejora educativa.

Para introducir el tema, abordamos cómo surgió la idea de dotar a las escuelas mexicanas de algún grado de autonomía; definimos el CTE, explicamos su misión y enumeramos los procesos que atiende.

La gestión escolar en México ha oscilado entre la centralización de la toma de decisiones y la transferencia de estas a los contextos locales. La Constitución de 1917 disponía que la función social educativa se distribuyera entre la Federación, los estados y los municipios (artículo $3^{\circ}$, fracción IX), pero la reforma constitucional de 1934 otorgó el poder de legislar, unificar y coordinar la educación de todo el país al Congreso y al Ejecutivo federal (Ornelas, 2013). En 1993 se modificó el artículo $3^{\circ}$ constitucional y se hicieron los cambios respectivos a la Ley General de Educación, mediante los cuales "se transfirió (a las entidades federativas) la operación de los servicios educativos federales (Fierro, Tapia García, Rojo y Directorate for Education OCDE, 2009); sin embargo, se fortaleció el papel protagónico del gobierno federal en las decisiones sustantivas del sistema educativo nacional.

En la década de los noventa, la atención de la autoridad educativa se centró en la escuela como unidad de cambio del sistema, "sus propuestas se refirieron básicamente a la necesidad de dar más libertad (autonomía) al equipo docente e incrementar su profesionalización como forma de obtener respuestas pedagógicas más 
acordes con las necesidades específicas de los alumnos y de su contexto" (Del Castillo y Azuma, 2009, p. 16). Así, el centro del cambio educativo se situó en el cruce de lo pedagógico con la organización y gestión.

A partir del ciclo escolar 2013-2014, el gobierno federal dispuso un cambio que reconocía la trascendencia de gestionar la educación desde lo local, al menos parcialmente, pues las decisiones importantes las siguió tomando la autoridad educativa federal. Los CTE se instituyeron como un espacio donde el colectivo docente "tiene el poder de transformar los centros escolares y generar los aprendizajes pertinentes y relevantes en los alumnos" (SEP, 2013, p. 7) a partir del trabajo colegiado, la autoevaluación y la toma de decisiones compartidas. La Secretaría de Educación Pública (SEP), con base en el acuerdo 15/10/17, los define como "el órgano colegiado encargado de tomar y ejecutar decisiones comunes enfocadas a que el centro escolar cumpla de manera uniforme y satisfactoria su misión" (SEP, 2013, p. 8); para ello, atiende tres procesos: la gestión de los procesos de enseñanza y aprendizaje en el aula; la gestión escolar; y la participación social en favor de los aprendizajes.

La autoridad educativa considera que el CTE velará por el desarrollo integral de los estudiantes al proporcionarles una educación de calidad. El director, profesores y supervisores serán corresponsables en este compromiso, trabajarán colaborativamente, compartirán el liderazgo y fortalecerán la autonomía de gestión en la escuela (SEP, 2013, p. 7).

A pesar del tiempo que tienen en operación los CTE, se han llevado a cabo pocos estudios que den cuenta de su funcionamiento y eficacia para cumplir con la finalidad planteada: fortalecer la autonomía de gestión, que permita aumentar la calidad de la educación al generar aprendizajes pertinentes y relevantes para los alumnos.

En las secciones siguientes presentamos una breve revisión de la literatura sobre liderazgo, el método seguido, los hallazgos sobre la conducción del CTE y las conclusiones.

\section{LIDERAZGO ESCOLAR}

En la literatura sobre el liderazgo se destaca el papel del director como agente de cambio, dado que es el vínculo entre las reformas que propone el Estado y la innovación ocurre al interior de las escuelas (Fend, 2011). En diversos países, el liderazgo escolar se ha convertido en una prioridad. De acuerdo con Pont, Nusche, Moorman y Hopkins (2008), este "juega un papel fundamental en la mejora escolar al influir en la motivación y capacidades de los profesores, así como en el clima escolar" (p. 9). De acuerdo con Robinson (2007), la evidencia señala que la mejoría en las escuelas no suele ocurrir si no hay un buen liderazgo. Según la OCDE (2004), hay una relación entre líder con mayor autonomía y los resultados en las evaluaciones PISA (Programa Internacional para la Evaluación de Estudiantes), pero esto no necesariamente se tendría que interpretar como una relación causal, pues ambas variables podrían depender de otros factores (Brauckmann \& Schwarz, 2014).

La concepción y el ejercicio del liderazgo han tenido cambios importantes desde los modelos individual, heroico y autoritario, hacia otros de carácter compartido, social y distribuido (Bush \& Glover, 2014); se considera una forma de influencia que se utiliza para inducir a otros a cambiar de manera voluntaria hacia el cumplimiento de tareas o 
proyectos propuestos por el líder (Bolívar, 1997). Congruente con esa idea, Bush (2017) señala que un líder escolar desarrolla una visión para sus escuelas y, a través de un proceso de influencia, hace que el grupo se mueva hacia al objetivo deseado. Además, el liderazgo incluye una gestión eficiente identificable con el rol de director y con una funcionalidad difusa de liderazgo moral entre los profesores (Bolívar, 1997). Este autor añade que, si el control burocrático y la autoridad técnico-racional no son suficientes o legitimables, se necesita incluir en el liderazgo "la autoridad moral y profesional, así como el acuerdo, la colegialidad y el liderazgo múltiple de los profesores" (p. 26).

El término liderazgo escolar es polisémico; la Organización de las Naciones Unidas para la Educación, la Ciencia y la Cultura (Unesco, 2016) lo define como "un proceso que identifica y guía el talento y la energía de los profesores, estudiantes y padres hacia el logro de objetivos educativos específicos" (p. 3). Este concepto no solo incluye el papel del director, sino también el de sus asistentes y otros miembros del personal, por lo cual el liderazgo puede estar distribuido (Unesco, 2016). Este organismo también reconoce la importancia de la distribución del liderazgo; sin embargo, en esta distribución no considera a los profesores.

Además del liderazgo formal del director, en las organizaciones escolares puede operar el liderazgo distribuido, que también es descrito en la literatura como compartido, colaborativo, democrático y participativo. La idea de distribución del liderazgo se puede confundir con facilidad con la distribución de responsabilidades administrativas (Leithwood, Louis, Anderson \& Wahlstrom, 2004). Fullan (2002) aboga por fomentar el liderazgo del profesorado, pues considera que ayuda al mejor funcionamiento de los centros escolares. El director debe contribuir a que los profesores asuman tareas de liderazgo a fin de desarrollar un liderazgo múltiple, al margen de la posición formal ocupada administrativamente.

En el liderazgo identificamos tres dimensiones comunes: influencia, visión y valores (Bush, 2011). La influencia está relacionada con el logro de los propósitos deseados, puede ser ejercida por una persona o, en el caso del liderazgo distribuido, por un grupo. La visión es fundamental, ya que esta constituye lo que los líderes proponen al personal y a otros para inducirlos a compartirla. Los valores son aquellas proposiciones morales sobre las cuales un líder fundamenta sus acciones personales y profesionales (Bush \& Glover, 2014).

El interés en el estudio del liderazgo académico o pedagógico se debe a que la calidad de este produce diferencias importantes en el aprendizaje de los estudiantes (Bush, 2016), aunque no es una variable que lo afecte de manera directa, sino que lo hace a través de su influencia en la práctica educativa de los profesores. Leithwood, Harris y Hopkins (2008) han encontrado vínculos fuertes entre el liderazgo eficaz y la mejora en las escuelas.

Diversos estudios han revelado que el liderazgo, después del efecto de los profesores, es la variable más importante en el aprendizaje de los estudiantes (Elmore, 2010; Leithwood, 2009; Murillo y Marcela, 2013). En palabras de Leithwood et al. (2004), "de entre los factores relacionados con la escuela que contribuyen al aprendizaje de los estudiantes en la escuela, el liderazgo es el segundo; sólo después de la enseñanza en el aula" (p. 3). Estos autores añaden que la evidencia señala que el liderazgo tiene un impacto de una cuarta parte de todos los factores que afectan el aprendizaje de los estudiantes. 
Las funciones principales del líder, de acuerdo con Leithwood et al. (2004), son: fijar el rumbo, fomentar el desarrollo del personal y rediseñar la organización. El impacto más importante del líder radica en fijar el rumbo, es decir, en ayudar a todos sus colegas a desarrollar una comprensión compartida acerca de las actividades y metas de la organización y de la necesidad de cumplirlas. El desarrollo del personal incluye ofrecer, entre otros elementos, estimulación intelectual, apoyo individualizado y modelos apropiados de buenas prácticas. El rediseño de la organización tiene que ver con los cambios en la estructura y la cultura escolar para facilitar el trabajo de los profesores.

No hay una sola forma de ser líder; Shukla y Bhagat (2013) plantean, de acuerdo con las ideas de Kurt Lewin, que hay siete tipos de liderazgo: autoritario, democrático, dejar hacer, transaccional, transformacional, servidor y carismático. Seguramente, en el contexto educativo, los más relevantes son el democrático y el transformacional, dado que, si se logra el involucramiento de todos y se les motiva a alcanzar el aprendizaje de los alumnos, apelando a la importancia que este tendrá en sus vidas, podrán originar cambios en la cultura escolar. Aunque por cuestión analítica los estilos se presentan separados unos de otros, en la realidad un líder puede mostrar características de más de un estilo.

Leithwood, Harris y Hopkins (2008, p. 3), en una revisión de la literatura, encuentran que, entre las múltiples revelaciones de las investigaciones acerca del liderazgo escolar, hay siete afirmaciones que están sustentadas en hallazgos empíricos, aunque en distinto grado:

-El liderazgo es el segundo factor más importante para el aprendizaje después de la enseñanza.

-Casi todos los líderes exitosos tienen el mismo repertorio básico de prácticas.

- Las maneras en que los líderes aplican estas prácticas básicas demuestran respuesta al contexto en que laboran, pero no están dictadas por este.

-Los líderes escolares mejoran la enseñanza de manera indirecta a través de su influencia en la motivación del personal, compromiso y condiciones laborales.

-El liderazgo escolar tiene una gran influencia en las escuelas y en los estudiantes cuando está ampliamente distribuido.

-Algunos patrones de distribución (del liderazgo) son más eficaces que otros.

-Una pequeña cantidad de características personales explican una gran proporción de las variaciones en la efectividad del liderazgo.

En el caso de la secundaria cuyo CTE fue observado, el liderazgo se muestra como uno de los aspectos principales del cual depende su buen funcionamiento. Por ello, analizamos las características del liderazgo tanto del director como de los profesores. Ambos aparecen en las sesiones de este órgano colegiado. 


\section{EL MÉTODO SEGUIDO}

Dada su naturaleza, el estudio se lleva a cabo dentro del paradigma cualitativo, ya que sus raíces en diversas disciplinas, dentro de las ciencias sociales, permiten abordar fenómenos sociales complejos (Marshall \& Rossman, 1995). Este paradigma ayuda a los investigadores a centrarse en procesos, obtener el punto de vista de los participantes, recolectar los datos en escenarios naturales y, sobre todo, comprender el fenómeno de manera inductiva (Cresswell, 2014) sin tener hipótesis preconcebidas para dar cuenta de lo que ocurre en las sesiones del consejo, primero de manera descriptiva y luego elaborando una explicación fundamentada de su funcionamiento.

Entre los métodos del paradigma cualitativo, elegimos el estudio de casos, ya que permite estudiar en profundidad fenómenos delimitados (Stake, 1999), y se interesa en incluir tanto los aspectos objetivos como subjetivos de la vida social (Neiman \& Quaranta, 2012). En palabras de Colina (2014), el estudio de caso es "una estrategia de investigación exhaustiva desde múltiples perspectivas de la complejidad y unicidad de un determinado proyecto, institución o sistema en un contexto real" (p. 245).

Las observaciones se hicieron en dos de las sesiones intensivas del CTE y en las reuniones ordinarias del ciclo 2016-2017 de una secundaria publica de la periferia de la ciudad de Guadalajara, Jalisco. Esta escuela fue elegida para el estudio porque, a juicio del supervisor, tiene un CTE eficaz. Además de las observaciones, se entrevistó en dos ocasiones a nueve de los profesores más participativos en el consejo y al director. Otra de las razones de haberlos elegido, más allá de su participación, es que eran profesores de planta y, por tanto, asistían a todas las reuniones del CTE y tenían mayor conocimiento de la escuela.

Los datos fueron codificados utilizando el software Atlas ti, se desplegaron en tablas y a partir de eso se llevó a cabo la descripción e interpretación. Para el análisis del liderazgo de los profesores y del proceso de influencia del director, utilizamos categorías inductivas; en el caso de los primeros, identificamos los tipos de actividad en que aparecía el liderazgo y las características de este; en el caso del director, reconocimos las maneras en que ejerce influencia en el colectivo docente mediante ciertas acciones específicas. En cambio, para el análisis de las funciones centrales y el tipo de liderazgo que despliega el director, recurrimos a las categorías provenientes de la teoría.

\section{RESULTADOS: ASÍ SE TRENZAN LOS HILOS}

Por disposición oficial, el director es quien dirige las sesiones del CTE (SEP, 2013). La dirección de la secundaria es de una alta complejidad porque se encuentra en la encrucijada de los intereses de la autoridad educativa a través de la supervisión escolar, el trabajo y los intereses de los profesores, las necesidades de aprendizaje concretos de los alumnos de la escuela y las demandas de los padres de estos. Todas estas demandas, intereses y necesidades diversas, que son como hilos que en esta escuela son trenzados por el líder formal, llegan al CTE y es ahí donde se procesan y se tejen; sin embargo, quienes fabrican los hilos son los profesores, pero ellos también participan en el trenzado más fino, en darle sentido a las actividades y a la información que se genera en la escuela. 


\section{Los hilos que fabrican los profesores}

Cuando se analiza el liderazgo en las escuelas, se tiende a pensar en lo que hacen los directores, de acuerdo con la literatura revisada, pero en el análisis de las interacciones en las sesiones del CTE, el liderazgo de los docentes también es fundamental. No se puede explicar el buen funcionamiento de este organismo colegiado sin esta conducción discreta, transitoria y, en ocasiones, múltiple que ejercen los profesores. Estos no colaboran en la planeación del trabajo del CTE, pero sí llevan sus experiencias al consejo, identifican necesidades, proponen soluciones, evalúan, diseñan el plan de trabajo, eligen las prioridades, trabajan en equipo, elaboran los productos que requiere la SEP, asumen liderazgos horizontales de manera temporal, reconocen y aceptan la autoridad del director.

Los profesores participan de manera diferente en las actividades del consejo, dependiendo de la índole de estas: encuadre, análisis, toma de decisiones y producción de documentos. En cada una de ellas hay diferentes maneras de participar.

Las actividades de encuadre son aquellas en las que el director proporciona información, por lo general en una presentación visual y, en otras ocasiones, en hojas impresas. En ellas comparte el objetivo de la sesión, los fundamentos teóricos y algunos conceptos. Los profesores tienen participaciones limitadas y muy pautadas por la planeación y la conducción del director; consisten en leer lo que aparece en la pantalla, por lo regular de manera individual, pero algunas veces lo hacen en coro. También se les solicita que expliquen lo que entienden por algún término, que manifiesten alguna opinión o elaboren un resumen sobre alguna idea que les fue expuesta. De este modo, se busca que haya una comprensión común de propósitos, conceptos centrales e información básica. La participación continua de los profesores, espontánea o solicitada, ayuda a que estos se centren en la actividad y a que resulte menos cansado; asumen un papel similar al de los estudiantes en el aula y no muestran iniciativas ni proactividad; participan de manera dócil y acatan todas las indicaciones.

En las actividades de análisis cambia el modo de participar de los profesores, pues en estas se organizan, asumen liderazgos temporales y compartidos, opinan, debaten, emiten juicios, revisan sus propias acciones, analizan los resultados de los alumnos y generan propuestas. En una de las sesiones, revisaron el número de horas que dedican a cada actividad en la escuela para determinar si se puede disminuir tiempo de algunas de ellas para dedicarlas a la enseñanza. Contaron las horas y debatieron para calcular meticulosamente el tiempo a fin de tener un panorama exacto (OBS_8). Este análisis no solo es útil para que la autoridad educativa se entere de lo que ocurre en la escuela, sino que también es un medio de control en que se evidencia lo que hacen como escuela y en lo individual. Esa transparencia permite tomar mejores decisiones. Mediante el ejercicio, resultó evidente que había información que no tenían clara; el CTE les permite recapitular lo que han hecho y planear con base en lo que ocurre. Sin el liderazgo de algunos profesores, este análisis no hubiera sido posible.

En las actividades de toma de decisiones, los profesores generan ideas, las debaten y las plasman en un objetivo, en un plan de trabajo, en la elección de un ámbito, en una estrategia de enseñanza o en un compromiso para la mejora escolar. No solo piensan colegiadamente qué es lo que conviene hacer, sino que, además, muestran evidencias de lo que hacen en el aula a partir de lo que decidieron en el consejo: 
Luego presenta algunas experiencias exitosas de actividades lúdicas. Presenta fotos en que se ve a los estudiantes aprendiendo las partes del discurso, las partes del cuerpo humano con una lotería, utilizando otras actividades didácticas como la pelota para [...] el paraguas para categorías de palabras. Dicen que usaron el pizarrón electrónico. Elige tu libro fue una actividad del profesor de matemáticas (Obs_5).

En las actividades de producción de documentos, los profesores elaboran carteles, llenan formatos o hacen algún escrito en que dan cuenta de su análisis, reflexiones y propuestas para la mejora escolar. Esta actividad es simultánea con las de análisis y de toma de decisiones, lo que la distingue es que consiste en la concreción de aquellas.

Los profesores se adaptan a cualquier modalidad de trabajo, individual, en parejas, en grupos al azar, en grupos por grado y en grupos por academia, lo que permite la interacción entre todos y contribuye a la cohesión del grupo.

Una característica destacable es la generosidad con la que se comparten materiales; un ejemplo es una lista de videos que están en internet para que los alumnos puedan comprender algunos temas de matemáticas. También se regalan entre ellos formatos de registro que han sido útiles para quien los ofrece; lo que más se comparten y solicitan son las actividades didácticas atractivas o lúdicas y que otro profesor ha diseñado y experimentado en su clase.

Cuando están en grupos pequeños y mediante la participación de todos, analizan las ideas y acuerdan qué incluir como producto de su trabajo; asumen liderazgos temporales y, en ocasiones, simultáneos; se muestran respetuosos, atentos y con frecuencia señalan que les ha sido muy importante tener este espacio de diálogo y trabajo con sus compañeros; en una ocasión, comentaron que en este tienen la posibilidad de interactuar con sus compañeros y aprender de los otros (OBS_6).

A diferencia del liderazgo del director, el de los profesores es menos notorio y ocurre de manera simultánea cuando se trabaja por equipos. Se caracteriza por ser horizontal y temporal. Cuando las actividades pasan del grupo grande a pequeños grupos, alguno de los participantes asume el liderazgo en forma espontánea. En otros casos, los profesores asignan a alguien la tarea de coordinar, por lo cual, de facto, se convierte en líder. En la tabla 1 presentamos las características del liderazgo de profesores.

Tabla 1. El liderazgo de los profesores

\begin{tabular}{|c|}
\hline Horizontal \\
\hline Dependiente de la actividad \\
\hline Asumido espontáneamente \\
\hline Reconocido por colegas \\
\hline Democrático \\
\hline
\end{tabular}

El liderazgo de los profesores dura el mismo tiempo que la actividad o puede ser muy corto, dado que otro miembro del equipo puede asumirlo en otra fase de la misma tarea. Es frecuente que haya liderazgos simultáneos, es decir, que uno de los profesores pida al equipo sus aportaciones mientras otro coordina la manera en que estas deben quedar plasmadas en un documento; en general, los profesores se muestran colaboradores y aceptan las directrices del director. En ocasiones, muestran puntos 
de vista diferentes que defienden con ahínco. Cuando son de índole cognoscitiva, es decir, ideas o maneras de presentar la información, el director cede, pero si se trata del cumplimiento de lo que establecen los reglamentos, el director señala lo que habrá de hacerse $y$, a final de cuentas, los profesores ceden.

Aunque el liderazgo de los profesores sea menos notorio y no esté legitimado por la autoridad, tiene una enorme importancia en el funcionamiento del CTE, pues es mediante esa conducción, cambiante, espontánea y aceptada por los otros, que se hace el trabajo fino de análisis, crítica y propuestas de mejora. Se precisan las características de ese liderazgo en tipos de actividades específicas sin la pretensión de afirmar que todo está bien hecho, sino de que el CTE no funcionaría bien sin el liderazgo de los docentes.

\section{El trenzado del director}

Trenzar todos los hilos, es decir, dar coherencia al trabajo a partir de los elementos que se generan durante las sesiones del CTE exige al director el desempeño de múltiples funciones: es anfitrión y se encarga de planear las sesiones junto con dos coordinadores docentes; reducir la información y actividades que plantea la SEP para las sesiones del CTE; preparar materiales y sistematizar datos; dar indicaciones; filtrar, mediar y resumir los diálogos de las sesiones; marcar los tiempos; regular el ritmo de trabajo; asegurar que se cumplan los objetivos que la autoridad plantea para cada sesión del CTE; señalar fallas a docentes en particular; reconocer aportaciones individuales o de grupo; fomentar un clima de trabajo agradable; y mantener el foco en los aspectos relevantes del análisis y las decisiones, entre muchas otras. En este escrito el centro está en las funciones de liderazgo.

\section{El proceso de influencia}

El análisis detallado del papel director devela las maneras específicas en que influye para que los profesores hagan modificaciones: funciona como anfitrión, media el aprendizaje, controla y mantiene el ritmo del trabajo. Además, critica y reconoce la labor de los profesores. En su función de anfitrión, da la bienvenida, muestra interés personal por los participantes, invita y provee el desayuno, agradece las participaciones, recibe y atiende a los visitantes, presenta a los nuevos profesores, fomenta un clima de grupo agradable, agradece el trabajo del día, invita al desayuno, y despide al grupo al terminar el trabajo.

El director también se encarga de controlar y mantener el ritmo del trabajo de los profesores para que no haya desfases entre los pequeños grupos y para que se alcancen los objetivos de cada sesión. En la tabla 2 compartimos algunas evidencias de cómo el director ejerce el control y mantiene el ritmo de trabajo. 
Tabla 2. Evidencias de atención al ritmo de trabajo y del control

\begin{tabular}{|c|c|}
\hline Ritmo de trabajo & Control \\
\hline $\begin{array}{l}\text { El director pregunta si ya terminaron le dicen que no } \\
\qquad\left(\mathrm{Obs}_{-} 1\right)\end{array}$ & $\begin{array}{l}\text { El director llama la atención a una profesora que se } \\
\text { acerca a otro equipo a hablar de otro tipo de asunto } \\
\text { (Obs_1) }\end{array}$ \\
\hline $\begin{array}{c}\text { El director dice de manera enfática que hay que llenar } \\
\text { eso. Que después podrán abordar el tema, ahora hay } \\
\text { que seguir con la actividad (Obs_3) }\end{array}$ & $\begin{array}{l}\text { Se pasea por el salón, dice “hasta acá se oye” porque } \\
\text { dos profesores hablan mientras todos los demás están } \\
\text { contestando el cuestionario (Obs_3) }\end{array}$ \\
\hline $\begin{array}{c}\text { 8:47 El director pregunta: “CCómo vamos?”. Se acerca a } \\
\text { la academia de matemáticas y pregunta si ya termina- } \\
\text { ron, le dicen que sí (Obs_3) }\end{array}$ & $\begin{array}{c}\text { Se acerca a los profesores mientras habla y recorre toda } \\
\text { la herradura. Pide a una maestra que lea los avances y } \\
\text { fortalezas que tiene la escuela (Obs_4) }\end{array}$ \\
\hline $\begin{array}{l}\text { Les dice que lo hagan rápido porque después tienen que } \\
\text { leer el concentrado por grado (Obs_4) }\end{array}$ & $\begin{array}{l}\text { Pide que anoten en la parte de atrás de las hojas los } \\
\text { nombres de los integrantes de los equipos (Obs_1) }\end{array}$ \\
\hline
\end{tabular}

El director asume el control de lo que ocurre en el consejo; para ello, planea, diseña materiales y actividades junto con el equipo directivo; critica a los profesores cuando, en su opinión, no están haciendo bien su trabajo, elogia, supervisa, anima y los apura para mantener el ritmo del trabajo para que, por una parte, no haya tiempos muertos por algunos de los profesores y, sobre todo, para alcanzar a cumplir con los objetivos en el tiempo establecido.

Un par de conductas relacionadas entre sí, que tiene el líder, son criticar y reconocer. Utiliza un tono de broma para cuestionar acciones que considera no conducentes a la formación de los alumnos y sonríe mientras lo dice. Por las sonrisas de los profesores, podemos inferir que no lo toman como agresión. Por otra parte, reconoce y elogia acciones concretas y, en ocasiones, características generales de los profesores.

En la tabla 3 mostramos algunas de las acciones de crítica y reconocimiento durante las sesiones del CTE.

Tabla 3. Evidencias de crítica, reconocimiento y elogio

\begin{tabular}{|c|c|}
\hline Critica & Reconoce y elogia \\
\hline $\begin{array}{c}\text { Les dice que están muy platicones, que quiere que se cen- } \\
\text { tren más en el trabajo (Obs_1) }\end{array}$ & $\begin{array}{c}\text { El director comenta que los ve tan involucrados que qui- } \\
\text { siera dejarles toda la mañana, pero no se puede (Obs_3) }\end{array}$ \\
\hline $\begin{array}{c}\text { Dice que fue bueno que no dio el tiempo para revisar evi- } \\
\text { dencias que porque no cree que las hayan traído. Dice que } \\
\text { hay que acabar la simulación (Obs_3) }\end{array}$ & $\begin{array}{c}\text { El director recalca que hacen buenos productos y que lo } \\
\text { sorprenden; luego, dice que en realidad no lo sorprenden, } \\
\text { pues él sabe el personal que tiene (Obs_4). }\end{array}$ \\
\hline $\begin{array}{c}\text { Llegan algunos profesores y él dice: “Corriendo y llegando } \\
\text { tarde" (Obs_4) }\end{array}$ & $\begin{array}{c}\text { El director les pide atención a lo que hicieron el conjunto } \\
\text { de mentes brillantes (Obs_4). }\end{array}$ \\
\hline $\begin{array}{c}\text { Dice que estuvo impactado por la falta de cumplimiento en } \\
\text { las academias en que trabajó con ellos (Obs_4) }\end{array}$ & $\begin{array}{c}\text { El director pide un aplauso para las recuperadoras y } \\
\text { para el equipo entero. Los profesores aplauden y sonríen } \\
\text { (Obs_4) }\end{array}$ \\
\hline
\end{tabular}

Con cierta frecuencia, el director, en tono de broma, señala a los profesores sus fallas y les pide que dejen de cometerlas, como: "[fulanito] debe de estar en el salón a las siete, no entrando a la escuela a esa hora"; "[zutanito] se trepa al escritorio, me dan ganas de quitarle el escritorio y dejarle solamente una silla"; "el profesor de tal materia es muy bueno, pero siempre llega tarde y a veces no viene"; "[fulanito] entrega las mismas planeaciones del año pasado, cuando menos debiera cambiarles la fecha".

El líder del consejo muestra una variedad de maneras de ejercer influencia entre los profesores. En su papel de anfitrión, genera un clima agradable de trabajo y atiende de modo personal a cada uno de los profesores. También, mantiene un ritmo de trabajo continuo y se asegura de que todos los docentes estén centrados en la tarea (control), critica las conductas docentes que en su opinión no contribuyen al trabajo y hace reconocimientos públicos de todas las aportaciones. 


\section{El liderazgo del director en el CTE}

En las secciones anteriores hemos presentado un análisis inductivo acerca del liderazgo de los profesores y la forma en que el director ejerce influencia entre el personal docente. En lo expuesto podemos notar que el liderazgo ejercido por unos y otro tiene características distintas (ver tabla 4).

Tabla 4. El liderazgo en el CTE

\begin{tabular}{|c|c|}
\hline Profesores & Director \\
\hline Horizontal & Vertical \\
\hline Dependiente de la actividad & Permanente \\
\hline Asumido espontáneamente & Atribuido por la autoridad \\
\hline Reconocido por colegas & Reconocido por los profesores \\
\hline Democrático & Democrático/autoritativo \\
\hline
\end{tabular}

Reconocemos que uno y otro muestran características diferentes, sin que ello implique un juicio de valor. Lo importante es identificar el papel de unos y otro en el funcionamiento del CTE. En otras secundarias, observamos liderazgos diferentes a los aquí descritos que dificultaban el trabajo de los consejos, pero que no analizamos en este artículo.

\section{Las funciones de liderazgo del director}

En lo que sigue, utilizamos categorías provenientes de la teoría para analizar el liderazgo del director. Examinamos las siguientes funciones de liderazgo que contribuyen al buen funcionamiento del consejo: fijar el rumbo, fomentar el desarrollo del personal y rediseñar la organización (Leithwood et al., 2004), así como el reconocimiento de los profesores a la manera en que el director conduce el trabajo.

Fijar el rumbo

Para fijar el rumbo, se requiere que el líder escolar tenga una visión de lo que considera deseable alcanzar (Bush, 2017). En el caso analizado, además, establece prioridades y pone el acento en los asuntos relevantes. Hay un momento en que el director declara la visión general:

El director dice que tienen como visión que están desarrollando competencias para la vida y contribuyendo al perfil de egreso. Pregunta a una profesora en dónde está eso, quien contesta que en las acciones. El director comenta que hay que tener claro a qué competencias le van a abonar desde cada asignatura. Dice que hay saturación de actividades y, por ello, a veces no saben qué abonan a las competencias (Obs_5).

No obstante, no se limita a este enunciado general, sino que lo reitera y desarrolla en distintos momentos del trabajo en varias de las sesiones, cuando declara que:

... la docencia no es para una generalidad sino para alumnos particulares (Obs_4);

Se trata de incluir y atender a la diversidad no a un alumno promedio abstracto (Obs_5);

Se trata de elevar los aprendizajes (Obs_6);

La evaluación no tiene que ver con entrega de trabajos, sino con el aprendizaje (Obs_8); 
No basta señalar las fallas sino de hacer propuestas valiosas (Obs_4);

Si no está dando resultado lo que hacen, ¿cuál es la propuesta? (Obs_5); Atendiendo el aprendizaje se fortalece la autoestima y motivación (Obs_5).

En distintos momentos, cuando los profesores hablan del entorno y de los papás como causa del bajo aprovechamiento y participación en clase de los alumnos, el director los centra preguntándoles cuál es el papel de la escuela, dado que eso es lo que está bajo su control. Es evidente que el director tiene una visión pedagógica y no psicologizante cuando señala que, a través del aprendizaje, se fortalecen la autoestima y la motivación, y no a la inversa. También, muestra su visión pedagógica al declarar que no hay alumno promedio abstracto, sino estudiantes concretos y que el objetivo es mejorar su aprendizaje.

El director considera a los grupos como un ente formado por una diversidad de personas y, por tanto, con una diversidad de necesidades que los docentes deben atender; que la evaluación ha de ser sumativa, es decir, dar cuenta del aprendizaje y no un número por la entrega de trabajos. Para mantener clara esta visión en el personal, acota la discusión a lo que la escuela puede hacer y evita, así, que los profesores expliquen los bajos resultados por factores relativos a la familia y al entorno.

De igual modo, el director delimita el análisis cuando los profesores se centran en las fallas de los estudiantes y los enfoca en la elaboración de propuestas para la mejora. Incluso, cuando la guía del CTE comenta que el profesor de un grado debe informar al que sigue acerca del desempeño de los estudiantes, el director, de manera enfática, precisa: "La recomendación no debe ser para predisponer a los siguientes profesores, debe ser la información que sirva para que ayuden mejor al alumno, no para que los etiqueten" (Obs_9).

La visión del director manifestada en lo que declara y en sus acciones en la conducción del CTE fija el rumbo, lo que constituye una forma de influencia en los profesores para que cambien sus ideas acerca de hacia dónde dirigir la labor docente. Esto se revela en algunas acciones de los profesores: hacer clases variadas y actividades lúdicas, "la pelota para [...], el paraguas para, lotería, elige tu libro, pizarrón electrónico, etc." (Obs_5), así como dejar trabajo diferenciado y atender la diversidad. Un ejemplo de esto último es que hay un niño con muchas dificultades para la expresión oral y escrita a quien le permiten tomar notas de la clase mediante dibujos y puede entregar tareas en la misma modalidad. Este proceso de influencia del líder ha inducido a los profesores a hacer cambios en sus prácticas, lo que concuerda con el planteamiento de Bush (2017), en el sentido de que el liderazgo influye en el aprendizaje de los estudiantes a través de la modificación de las acciones de los docentes.

Como advertimos, las evidencias provenientes de las observaciones y las entrevistas muestran que el director orienta el rumbo en el CTE, función clave de un líder.

Fomentar el desarrollo del personal

La segunda función del director que señalan Leithwood et al. (2004) es fomentar el desarrollo del personal, la que también cumple el director de nuestro estudio. La manera más obvia de promoverlo es mediante la invitación a cursos, por ejemplo, 
en la tercera reunión intensiva del CTE los alienta a que asistan a un curso de Google en sábado para que aprendan a entregar documentos en versión electrónica. En total, 12 personas le pidieron que los inscribiera. También hay otras maneras en que lo procura, al mediar el aprendizaje, llevar las ideas a la práctica, concretar ideas abstractas, facilitar que se compartan las experiencias exitosas, dar sentido a las prácticas y ayudar a la generación de conocimiento pedagógico. En una de las sesiones, muestra una aspiradora elaborada por un alumno con material reciclado como ejemplo de la concreción del aprendizaje en un producto físico (Obs_4). Esto concuerda con el señalamiento de Leithwood (2004) de que el desarrollo del personal incluye la estimulación intelectual y el modelamiento de buenas prácticas.

El director fomenta el desarrollo profesional de los docentes ayudándoles a precisar o ampliar su pensamiento, o bien, a aplicar lo pensado a su práctica profesional y a proporcionar evidencias, y sobre todo disponiendo de lo necesario en las sesiones del CTE para que los profesores identifiquen problemas concretos, desarrollen propuestas viables y muestren evidencias de su aplicación en el aula; a esto le podemos llamar mediación del aprendizaje (más adelante presentamos la evidencia).

El proceso de fomentar el desarrollo profesional se entiende como la acción del director de colocarse entre los profesores como aprendices y su objeto de aprendizaje (Parra y Keila, 2014), que en este caso es el conocimiento pedagógico. En esta categoría, las acciones son muy diversas: plantear con claridad los objetivos; estructurar el trabajo; procurar la comprensión de los profesores; proporcionar información; resumir las aportaciones y vincularlas con el objetivo de la sesión; dar indicaciones; alentar la participación; cuestionar a los docentes; marcar los tiempos para las actividades; mantener el foco en que el objetivo de todo es que los alumnos aprendan; y manejar el flujo de información entre todos los actores involucrados.

Otra de las formas en que se presenta la mediación es la ayuda para concretar las ideas abstractas. Ante la pregunta, ¿qué significa garantizar el derecho de los niños y jóvenes a la educación?, los profesores dan respuestas abstractas, cercanas a las disposiciones oficiales: artículo tercero, calidad, educación obligatoria, etcétera. El director se acerca al equipo y ayuda a que concreten la idea en que "se requieren buenos maestros" (Obs_1). Otro ejemplo de mediación es que les ayuda a que completen las ideas. En un equipo, los profesores proponen que se deben replantear las acciones para el logro de objetivos y metas para la ruta de mejora: "El director pregunta para qué, le contestan que para el logro de los aprendizajes clave, el director pregunta de quién y le dicen que de los educandos" (Obs_1). Así, es completada la idea de que se deben replantear los objetivos y las metas para el logro de los aprendizajes clave de los alumnos.

En ocasiones, los ayuda a encontrar razones para las acciones que emprenden, por ejemplo, "pide a los profesores que argumenten por qué eligieron ciertas estrategias si no eran las que ellos habían diseñado" (Obs_3); o les formula preguntas para que vayan más allá de los datos, como "¿qué formas de trabajo se requieren para mejorar el aprendizaje?” (Obs_1) o “por qué pueden pensar que educación física no tiene nada que ver?” (Obs_4), o bien, “¿a qué atribuyen que no haya funcionado?” (Obs_5).

También, apoya la formación profesional aportando materiales. En una de las sesiones les comenta que pueden compartir experiencias de estrategias de aprendizaje exitosa y se asegura de que, independientemente de quién las haya utilizado, las 
tengan todos los profesores: "El director dice que van a tomarle una foto a las estrategias para que las tengan todos" (Obs_3); de igual modo, les pide las evidencias de que están cumpliendo en las aulas lo que acuerdan en el consejo. En las notas de campo se da cuenta de ello:

Luego presenta algunas experiencias exitosas de actividades lúdicas. Presenta fotos en que se ve a los estudiantes aprendiendo las partes del discurso, las partes del cuerpo humano con lotería utilizando otras actividades didácticas como la pelota para [...] el paraguas para categorías de palabras. Dicen que usaron el pizarrón electrónico. Elige tu libro fue una actividad del profesor de matemáticas (Obs_5).

Esta manera de conducir las sesiones del CTE y las intervenciones del director ayudan a los profesores a dar sentido a ciertas prácticas, como lo manifestó una profesora:

Cuando se empezó a implementar el CTE, muchos compañeros vimos que la planeación era importante porque nos iba a ayudar en el aula a mejorar la atención a los grupos y centrar lo que se estaba haciendo en el temario de la materia. Así que, cuando siguió esa parte de que la planeación era una parte importante, se disparó, haz de cuenta que la mayoría de las personas empezó a entregar sus planeaciones y se empezó a centrar más el trabajo académico en ese sentido (Ent_4).

Las acciones del director fomentan el desarrollo de los profesores al empujar a que se genere un conocimiento pedagógico, no como teoría, sino como práctica. Uno de ellos señala: "Intercambiamos experiencias, formas de evaluar, eso es lo que hacemos [...] con compañeros de diferentes áreas" (Ent_1). De esta manera, fomenta el mejoramiento de la práctica, el desarrollo profesional y, a través de eso, se incrementa el potencial para mejorar la atención a los estudiantes.

\section{Rediseñar la organización}

Esta es la función de liderazgo que se percibe con más limitaciones, pues el margen para hacer modificaciones es pequeño. Por una parte, hay una regulación muy amplia por parte de la SEP, como, por ejemplo, no suspender ni una sola hora de clase, lo que impide que los profesores puedan reunirse para planear y pensar nuevas maneras de realizar el trabajo o atender a ciertos alumnos con requerimientos especiales; por otra, los recursos son pocos. Para la compra de materiales y cubrir gastos básicos de la escuela, dependen de las aportaciones voluntarias de una parte de los padres de los estudiantes y de la venta de alimentos a los alumnos; por tanto, tienen que limitar hasta el número de copias que un profesor puede solicitar (Obs_9).

Aunado a lo anterior, hay una gran cantidad de horas no cubiertas porque la Secretaría de Educación Jalisco no envía profesores. En el ciclo 2015-2016 no se cubrían 127 horas de clase por semana en el turno vespertino y 49 en el matutino (Obs_2). Una carencia más es la falta de suficientes profesores de planta. Hay profesores que solo tienen cuatro horas de clase a la semana y se trasladan continuamente de un municipio a otro debido a que trabajan en diversas escuelas (0bs_1).

Ante estas condiciones, las transformaciones que se pueden hacer son pequeñas, limitadas a algunas acciones administrativas y pedagógicas. Una de las que observamos fue la implementación de un programa para reducir el absentismo y la deserción. El absentismo es de entre el 5\% y el 13\% diario. No todos los días faltan los 
mismos alumnos; el viernes asisten menos (Obs_1). Un absentismo del 13\% significa que en un día pueden faltar 182 alumnos. Para atender el problema, acordaron que todos los profesores registrarían con cuidado la asistencia para que Asistencia Educativa hiciera visitas domiciliarias cada que un mismo alumno faltara tres veces (Ent_9). Además de estas acciones, se pensó en ofrecer actividades en línea para quienes no pudieran asistir.

Para mejorar el aprendizaje de los alumnos, se intenta hacer una transformación pedagógica al incluir actividades lúdicas con el objetivo de involucrar más a los estudiantes en las actividades de aprendizaje e incidir en la motivación para que se incremente la asistencia. En las sesiones del CTE se presentan las actividades lúdicas diseñadas por los profesores, como el uso creativo de la biblioteca (Obs_6), el globo aerostático y las biografías de inglés (Obs_7), el paraguas de matemáticas (Obs_4), el prototipo de barredora eléctrica elaborada por uno de los estudiantes (Obs_9), entre otras. Incluso, procuran que procesos básicos como comprensión lectora y matemáticas se promuevan en las diversas asignaturas. Por poner solo un ejemplo, en la clase de educación física una de las actividades consistió en que los alumnos se tomaran de la mano y uno quedara en el centro mientras el grupo giraba y el del extremo externo, a manera de compás, dibujaba un enorme círculo en el piso del patio. Asimismo, se pusieron en práctica acciones para mejorar la convivencia y otras para atender de manera diferenciada a estudiantes con dificultades en el aprendizaje.

De las tres funciones del liderazgo, es claro que el director ha fijado el rumbo pedagógico, ha estimulado el fomento del aprendizaje, la atención a la diversidad, el uso de la evaluación como medio para aprender y la solución de los problemas desde lo pedagógico. También, ha impulsado el desarrollo del personal al mediar el aprendizaje de los profesores, allegar recursos y organizar el trabajo. En menor medida, ha promovido el rediseño de algunas actividades de la organización.

A pesar de los esfuerzos de los profesores y de un buen liderazgo, tanto de los profesores como del director, lo que puede hacer el CTE está restringido por la imposición central, por la gran cantidad de trabajo que requieren hacer para elaborar los productos que se tienen que entregar y porque la atención de la autoridad sigue estando en el control, lo que provoca una paradoja: se quiere fomentar la autonomía a través del control. Por tal motivo, el director considera que no es mucho lo que se logra con el CTE, pues, en su opinión, se tienen que hacer demasiados informes; algunos profesores simulan modificar su práctica, pero trabajan tanto como pueden para alcanzar mejores aprendizajes, atender a los niños más vulnerables y disminuir la deserción y el absentismo (E_9).

\section{El tipo de liderazgo}

Con base en los siete tipos de liderazgo más comúnmente citados en la bibliografía sobre el tema (Shukla \& Bhagat 2013), el del líder de esta escuela no tiene características de laissez faire, de transaccional ni de servidor, pero sí de los otros cuatro: cuando se trata de la normativa escolar, asume un estilo autocrático, es decir, fundamentado en el poder de su puesto como director; por ejemplo, ante la pregunta sobre la posibilidad de expulsar a un niño, responde: "Nada puede ir contra el derecho de los niños a la educación" (Obs_10). En otra ocasión, ante la necesidad de cuidar la 
integridad física de los niños y las niñas, el director dispone que algunos profesores tengan que hacer guardias durante el receso, ya que ese tiempo les es pagado. Ante la queja de los profesores, argumenta que no pueden culparlo por lo que ordena la autoridad, pero complementa con un elemento de liderazgo transformacional: "Les dice que tiene que ser la mejor escuela y que se requiere su apoyo" (Obs_8).

Cuando las disposiciones de la autoridad inmediata contravienen la legislación educativa, el director se atiene a esta última y no impone los criterios de la supervisión. En una de las sesiones, el supervisor afirma que el artículo quinto de un reglamento especifica que los profesores de tiempo variable deben asistir al consejo, aunque no sea dentro de su tiempo contratado; posteriormente, el director señala que ningún reglamento está por encima de la Ley Federal del Trabajo y que no tienen por qué venir, sino en el horario contratado (Obs_2). Cuando su liderazgo se torna autocrático, es para mantener el orden y el cumplimiento en el trabajo, y también para proteger los derechos de los profesores.

En otros momentos, el liderazgo del director es democrático. Aunque dirige el consejo, cuando existen diferencias de opinión entre él y el colectivo docente, prevalece la opinión de este. En la sesión de mayo se dialogó en torno a si harían un examen diagnóstico para toda la escuela o por turnos; después de discutirlo, acordaron que sería uno por turno (Obs_8). En la cuarta sesión intensiva aparece con mayor frecuencia la toma de decisiones de los profesores. Durante el trabajo se propusieron cuatro formulaciones del objetivo, se sometió a votación y eligieron la cuarta (Obs_2). Posteriormente, entre distintas opciones de metas, cambiaron de opinión y se decidieron por la tercera; el director había aceptado la primera, pero al final admitió la que prefirió la mayoría.

Un ejemplo más de liderazgo democrático se advierte en la tercera reunión intensiva cuando el colectivo docente no alcanzó a terminar la estrategia global, pues tenían cuatro propuestas, una por cada mesa de trabajo. El director pidió autorización para que él, junto con los coordinadores, redactara una que reflejara el trabajo de todos. Los profesores aceptaron (Obs_1).

El colectivo docente puede tomar decisiones y opinar libremente acerca de los asuntos que tratan. Cuando hay desacuerdos, prevalece la decisión de los profesores cuando estas son atribuciones del consejo, pero si son de índole administrativa o legal, el director determina lo que habrá de hacerse. Los dos ámbitos son reconocidos por los participantes y consienten que una de las partes tome las decisiones.

La mayor parte del tiempo, el liderazgo que asume es transformacional, pues intenta motivar a los profesores mediante la idea de que lo más importante es que los estudiantes aprendan y que ninguno debe quedar sin ser atendido, como se ha mostrado cuando se presentó la visión. De acuerdo con la literatura, el liderazgo transformacional es eficaz para mejorar los resultados de las escuelas (Leithwood et al., 2004; Oyentunji, 2006), y funciona para afrontar ambientes complejos y dinámicos, como los escolares.

Finalmente, cuando el director crea un clima de respeto, de cercanía afectiva, cuando escucha y reconoce la valía de las aportaciones, su liderazgo tiene elementos del liderazgo carismático, como en las siguientes situaciones: en la tercera sesión intensiva, él pregunta a un profesor sobre el resultado de sus análisis médicos, en 
otra "va saludando por su nombre a diferentes personas, les hace algún comentario como “tenía mucho tiempo que no te veía”' (Obs_6); en una más, llega una profesora y "el director la abraza y le dice que la estaban extrañando" (Obs_3). No solo muestra cercanía con los profesores que tienen tiempo en la escuela, sino también con los nuevos: cuando llegó un profesor muy joven que impartiría inglés en esa escuela, él le dijo: "Enseguida te presento, pequeño" (Obs_1). En otros momentos, elogia al grupo completo. En la tercera reunión ordinaria, "el director les pide atención a lo que hicieron en conjunto de mentes brillantes" (Obs_4) o muestra señalas de cuidado: "Pongan atención mis hijos, porque si no, nomás uno se está desgastando" (Obs_8).

De igual modo, muestra este tipo de liderazgo cuando elogia a los profesores al decirles que "tienen ellos una gran riqueza de conocimiento, varios sonríen" (Obs_4) o que "desde al inicio había comentado que no era necesario un trabajo que les pedían, porque ellos ya habían analizado la información. Sabía que tenían profesores de primera, muy responsables" (Obs_8).

El liderazgo, en la organización escolar, no es de un tipo definido, sino que presenta características de varios: autoritario, democrático, transformacional y carismático, pero también es antitético a otros, pues en ningún momento observamos que el director dejara hacer, que tuviera una línea de mando establecida, ni que estuviera en función de las necesidades de los profesores.

\section{El reconocimiento del grupo}

El liderazgo que despliega el director no es solo funcional; los profesores le reconocen lo que Bolívar (1997) llama un liderazgo moral. Durante las entrevistas con algunos profesores que participan en el CTE, apareció este como elemento primordial del buen funcionamiento de ese organismo colegiado, quizá más importante que el ejercicio de la autoridad. Esto se muestra en los siguientes extractos de las entrevistas:

No me pide algo que ella no sea capaz de dar (Ent_3);

Yo le reconozco al director muchas cosas. Es una persona muy tenaz, muy organizada (Ent_2);

El mismo día del año pasado estaba pesadísimo. Salía arrastrándose prácticamente, pero él estaba al pie del cañón [...] Recuerdo en una ocasión que se fracturó el pie. Lo traíamos en silla de ruedas aquí a la escuela;

Yo siento que él le echa muchas ganas a los consejos. Sí prepara todo (Ent_5); Tiene un liderazgo excepcional. Tiene un conocimiento de normatividades y demás que mis respetos (Ent_3);

[los desacuerdos] se resuelven de manera democrática [...] se toma en consideración el resto, el colectivo, que es lo que dice la mayoría del colectivo (Ent_4);

Es muy importante también que se escuchan las voces en la pluralidad de pensamiento, la diversidad de ese pensamiento (Ent_4);

Algunos compañeros sin respeto al director [habla de otra escuela] y yo digo mmm aquí no se da eso (Ent_2). 
Los profesores reconocen ciertos atributos personales del director, entre otros, la congruencia entre lo que pide y lo que hace, su organización y tenacidad, su esfuerzo, su conocimiento, la escucha y la manera democrática de tomar decisiones. Estas características, al parecer, permiten que los profesores acepten su liderazgo.

Además de los atributos personales mencionados, los profesores le reconocen al director ciertas acciones de liderazgo:

Hay mucha comunicación [...] el director la propicia (E_5);

El director nos incita mucho a que participemos (Ent_7);

Una de las cosas de su liderazgo es que delega, otra cosa es que no suelta la dirección y otra cosa es que involucra a varios en la reflexión de algo (E_3);

En una ocasión, el inspector llegó y pasó como Juan por su casa y se puso a hacer observaciones de clase. Entonces posterior, él le dice: ¿va a pasar? Ok, pero debo estar informado yo (E-3).

Uno de los entrevistados, el más crítico en las reuniones del CTE, declara:

Sin embargo, yo veo aquí que sí hay un liderazgo transformacional de alguna forma a decirlo ¿no? En el que se escuchan las opiniones de los compañeros. En el caso del director de esta escuela pues vemos que sí hace énfasis en la participación. Nos piden participar a todos, nos trae participando de manera activa a todo el personal. Se recogen las opiniones de los equipos, de los grupos de participación y creo que por lo menos aquí, ese liderazgo transaccional está transitando a un liderazgo transformacional. Eso es importante, o sea, es muy importante ese liderazgo. Si él logra un liderazgo de ese tipo al 100\% se van a ver resultados favorables en la escuela (Ent_4).

Este liderazgo moral, que consiste en dirigir a todo el personal hacia metas socialmente deseables, por parte de un profesional preparado, congruente en sus acciones con lo que declara, que promueve la colegialidad y la búsqueda de acuerdos, hace posible que los profesores se involucren en la toma de decisiones y, en alguna medida, en la puesta en acción de algunas de ellas. Por otra parte, ellos también asumen el liderazgo en ciertos momentos del trabajo.

\section{CONCLUSIONES}

Uno de los factores clave para el buen funcionamiento del CTE es el liderazgo, ya que tanto el del director como el de los profesores trenzan los hilos del tejido escolar durante las sesiones del consejo. Procuran no dejar cabos sueltos y asegurar que se atiendan, en la medida de lo posible, las diversas necesidades que presenta una organización tan compleja como la escuela. La revisión cuidadosa de los datos empíricos sirvió de evidencia de que, en una organización compleja, existen liderazgos múltiples y que el de los profesores, aunque menos visible, es tan importante como el del director. Uno y otro tienen propósitos y características distintas.

En el análisis del liderazgo de los profesores, más allá de reconocer sus características específicas: horizontalidad, dependiente de la actividad, temporal, asumido espontáneamente, etcétera, identificamos las actividades en las cuales lo ejercían: análisis, toma de decisiones y elaboración de documentos. Por otra 
parte, en las actividades de encuadre no se manifestaba ningún liderazgo por parte de ellos, pues reaccionaban a las instrucciones del conductor de la sesión de manera individual.

Estos liderazgos que ocurren sin invadir los campos de acción de unos y otros logran construir un ambiente propicio para el trabajo, con metas y focos claros, con la participación de todos los miembros del consejo y en un clima de trabajo capaz de atender las necesidades de la escuela, producir trabajo conjunto y resolver las fricciones que ocurren en las sesiones durante el ciclo escolar.

La intención de este estudio fue generar conocimiento acerca de uno de los factores principales del funcionamiento del CTE. Los hallazgos reportados pueden contribuir a que directores y colectivos docentes de otras escuelas revisen las características de los liderazgos de este caso para que consideren la posibilidad de aprender de ellos y, así, mejorar el desempeño de sus propios consejos.

\section{REFERENCIAS BIBLIOGRÁFICAS}

Bolívar, A. (1997). Liderazgo, mejora y centros educativos. En A. Medina (coord.). El liderazgo en educación (pp. 25-46). Madrid: UNED.

Brauckmann, S. \& Schwarz, A. (2014). Autonomous leadership and a centralised school system: An odd couple? Empirical insights from Cyprus (vol. 28). https:// doi.org/10.1108/IJEM-08-2013-0124

Bush, T. (2017). Lo educativo en la escuela. Nueve miradas. En Liderazgo escolar: perspectivas globales. Chile: Ediciones Universidad Diego Portales. Recuperado de http://cedle.cl/wp-content/uploads/2018/03/Liderazgo-Educativo-en-la-Escuela.-Nueve-miradas.pdf

Bush, T. (2016). School leadership and management in England: The paradox of simultaneous centralisation and decentralisation. Research in Educational Administration \& Leadership, vol. 1, núm. 1, pp. 1-23. Recuperado de http:// eprints.nottingham.ac.uk/35422/1/REAL\%20JOURNAL\%20ARTICLE\%20 APRIL\%202016\%20\%28Bush\%29.pdf

Bush, T. (2011). Succession planning in England: New leaders and new forms of leadership. School Leadership \& Management, vol. 31, núm. 3, pp. 181-198. https://doi.org/10.1080/13632434.2010.545383

Bush, T. \& Glover, D. (2014). School leadership models: What do we know? School Leadership \& Management, vol. 34, núm. 5, pp. 553-571. https://doi.or $\mathrm{g} / 10.1080 / 13632434.2014 .928680$

Colina, A. (2014). El estudio de caso, una estrategia para la investigación educativa. En Á D. Barriga y L. M. Bertha (2014). Metodología de la investigación educativa aproximaciones para comprender sus estrategias. Tlaxcala: Universidad Autónoma de Tlaxcala.

Cresswell, J. (2014). Research design: Qualitative, quantitative and mixed methods approaches (4a ed.). Thousand Oaks: Sage.

Del Castillo, G. y Azuma, A. (2009). La reforma y las políticas educativas. Impacto en la supervisión escolar. México: Flacso.

Elmore, R. (2010). Mejorando la escuela desde la sala de clases. Santiago, Chile: Fundación Chile. Recuperado de http://www.eduglobal.cl/download/Eduglobal31.pdf 
Fend, H. (2011). Die Wirksamkeit der Neuen Steuerung -Theoretische und methodische Probleme ihrer Evaluation. Zeitsch rift für Bildungsforschung, vol. 1, pp. 5-24. Recuperado de http://www.forschungsnetzwerk.at/downloadpub/fend_neue\%20steuerung_fend.pdf

Fierro, M., Tapia García, G., Rojo, F. y Directorate for Education OCDE (2009). Descentralización educativa en México: un recuento analítico. OCCDE. Recuperado de https://www.oecd.org/mexico/44906363.pdf

Fullan, M. (2002). Las fuerzas del cambio. Explorando las profundidades de la reforma educativa. Madrid: Akal.

Leithwood, K. (2009). Cómo liderar nuestras escuelas: aportes desde la investigación. Santiago, Chile: Fundación Chile. Recuperado de http://ww2.educarchile.cl/UserFiles/P0038/File/libros/Libro_Liethwood.pdf

Leithwood, K., Harris, A. \& Hopkins, D. (2008). Seven strong claims about successful school leadership. School Leadership \& Management, vol. 28, núm. 1, pp. 27-42. https://doi.org/10.1080/13632430701800060

Leithwood, K., Louis, K. S., Anderson, S. \& Wahlstrom, K. (2004). How leadership influences student learning. Nueva York: The Wallace Foundation.

Marshall, C. \& Rossman, G. (995). Designing qualitative research. EUA: Sage.

Murillo, F. J. y Román, M. (2013). La distribución del tiempo de los directores y las directoras de escuelas de educación primaria en América Latina y su incidencia en el desempeño de los estudiantes. Revista de Educación, vol. 361, pp. 141-170.

Neiman, G. \& Quaranta, G. (2012). Los estudios de caso en la investigación cualitativa. En Estrategias de investigación cualitativa. Barcelona: Gedisa.

OCDE (2004). Raising the quality of educational performance at school (policy brief). París.

Ornelas, C. (2013). El sistema educativo mexicano. La transición de fin de siglo. México: Fondo de Cultura Económica.

Oyentunji, C. 0. 2006. The relationship between leadership style and school climate in Botswana Secondary Schools. Tesis de doctorado. University of South Africa.

Parra, F. \& Keila, N. (2014). El docente y el uso de la mediación en los procesos de enseñanza y aprendizaje. Revista de Investigación, vol. 38, núm. 83, pp. 155-180. Recuperado de http://www.scielo.org.ve/scielo.php?script=sci_ abstract\&pid=S1010-29142014000300009\&lng=es\&nrm=iso\&tlng=es

Pont, B., Nusche, D., Moorman, H. \& Hopkins, D. (2008). Improving school leadership. París: OCDE.

Robinson, V. (2007). The impact of leadership on student outcomes: Making sense of the evidence, vol. 6. Recuperado de https://research.acer.edu.au/cgi/ viewcontent.cgi?article $=1006 \&$ context=research_conference_2007

SEP (2013). Lineamientos para la organización y el funcionamiento de los consejos técnicos escolares. Educación básica. México.

Shukla, A. \& Bhagat, S. (2013). Dynamics of creative leadership in modern context. An Analytical View, vol. 2, núm. 6, pp. 2319-2828. https://doi.org/doi.10.2139

Stake, R. (1999). Investigación con estudio de casos. Madrid: Morata.

Unesco (2016). Leading better learning: School leadership and quality in the Education 2030 agenda. Recuperado de http://www.unesco.org/new/fileadmin/MULTIMEDIA/HQ/ED/pdf/leadership-report.pdf 